\title{
Energy Efficiency Optimization for Heterogeneous Cellular Networks
}

DOI:

10.1109/VTCSpring.2017.8108465

\section{Document Version}

Accepted author manuscript

Link to publication record in Manchester Research Explorer

\section{Citation for published version (APA):}

Tang, J., So, K. C., Alsusa, E., Hamdi, K., Shojaeifard, A., \& Wong, K-K. (2017). Energy Efficiency Optimization for Heterogeneous Cellular Networks. In IEEE Vehicular Technology Conference Spring 2017 IEEE.

https://doi.org/10.1109/VTCSpring.2017.8108465

\section{Published in:}

IEEE Vehicular Technology Conference Spring 2017

\section{Citing this paper}

Please note that where the full-text provided on Manchester Research Explorer is the Author Accepted Manuscript or Proof version this may differ from the final Published version. If citing, it is advised that you check and use the publisher's definitive version.

\section{General rights}

Copyright and moral rights for the publications made accessible in the Research Explorer are retained by the authors and/or other copyright owners and it is a condition of accessing publications that users recognise and abide by the legal requirements associated with these rights.

\section{Takedown policy}

If you believe that this document breaches copyright please refer to the University of Manchester's Takedown Procedures [http://man.ac.uk/04Y6Bo] or contact uml.scholarlycommunications@manchester.ac.uk providing relevant details, so we can investigate your claim.

\section{OPEN ACCESS}




\title{
Energy Efficiency Optimization for Heterogeneous Cellular Networks
}

\author{
Jie Tang ${ }^{1}$, Daniel K. C. So ${ }^{2}$, Emad Alsusa ${ }^{2}$, Khairi Hamdi ${ }^{2}$, Arman Shojaeifard ${ }^{3}$ and Kai-Kit Wong ${ }^{3}$ \\ ${ }^{1}$ South China University of Technology, Email: eejtang@scut.edu.cn \\ ${ }^{2}$ University of Manchester, Email: \{d.so, e. alsusa, k.hamdi\}@manchester.ac.uk \\ ${ }^{3}$ University College London, Email: \{a.shojaeifard,kai-kit.wong\}@ucl.ac.uk.
}

\begin{abstract}
In this paper, we provide joint subcarrier assignment and power allocation schemes for quality-of-service (QoS)constrained energy-efficiency (EE) optimization in the downlink of an orthogonal frequency division multiple access (OFDMA)based two-tier heterogeneous cellular network (HCN). Considering underlay transmission, where spectrum-efficiency (SE) is fully exploited, the EE solution involves tackling a complex mixed-combinatorial and non-convex optimization problem. With appropriate decomposition of the original problem and leveraging on the quasi-concavity of the $\mathrm{EE}$ function, the problem can be efficiently solved. On the other hand, the inherent intertier interference from spectrum underlay access may degrade EE particularly under dense small-cell deployment and large bandwidth utilization. We therefore develop a novel resource allocation approach based on the concepts of spectrum overlay access and resource efficiency (RE) (normalized EE-SE tradeoff). Specifically, the optimization procedure is separated where the macro-cell optimal RE and the corresponding bandwidth is first determined, then the $\mathrm{EE}$ of small-cells utilizing the remaining spectrum is maximized. Simulation results confirm the theoretical findings and demonstrate that the proposed resource allocation schemes can approach the optimal $E E$ with each strategy being superior under certain system settings.
\end{abstract}

\section{INTRODUCTION}

The global mobile data traffic, thanks largely to the evergrowing use of applications on smart devices, increased by a tremendous $4 \mathrm{k}$ times in a decade from 2005 to 2015 and is expected to further grow going into 2020 and beyond. It is well-understood that the conventional cellular network architecture using macro-cells only cannot possibly support demand going forward. This trend has driven the wireless industry to devise new technologies and standards for a new fifth-generation $(5 \mathrm{G})$ mobile network. A promising enabler for supporting user equipments (UEs) with increased density and quality-of-service (QoS) requirements is to deploy different types of base stations (BSs), thus forming what is referred to as heterogeneous cellular network $(\mathrm{HCN})$ [1]. The underlying air interface technology for $\mathrm{HCN}$ in the downlink is orthogonal frequency division multiple access (OFDMA) as specified in modern cellular standards [2].

Meanwhile, spectrum-efficiency (SE), a measure of the total amount of information transmitted per unit bandwidth, has been used as a key performance indicator in the design and analysis of cellular networks [3], [4]. On the other hand, placing the focus solely on maximizing SE will lead to everrising network power consumption, which goes against global commitments for sustainable development jointly in terms of energy cost and environmental factors. Energy-efficiency (EE), defined as the total amount of information delivered per unit energy, is widely recognized as an important measure for joint spectrum- and energy-efficient cellular network design. The EE optimization problem has attracted great interest in the context of OFDMA-based HCNs [5]-[7].

In contrast to the previous works in [5]-[7] where the impact of spectrum utilization is not considered, in this paper we include bandwidth usage in the analysis by modeling the dynamic circuit power consumption as a linear function of the bandwidth [8]. As a result, the global frequency reuse strategy may lead to higher circuit power consumption and degraded EE performance. On the other hand, utilizing overlay transmission, where the allocated bandwidth for the macro-cell and the small-cells are exclusive, is considered a promising strategy when it comes to densely deployed or bandwidthabundant HCNs. In this paper, a fundamental study of EE in the context of an OFDMA-based two-tier HCN consisting of a macro-cell and multiple small-cells is provided. We consider both underlay and overlay transmission strategies and provide resource allocation schemes for maximizing $\mathrm{EE}$ subject to satisfying QoS constraints.

\section{PRELIMINARIES}

We consider the downlink of an OFDMA-based two-tier HCN comprising of a macro-cell and $L$ small-cells. The set of cells is denoted using $\mathcal{L}=\{0,1,2, \cdots, L\}$, where indexes 0 and $\{1,2, \cdots, L\}$ correspond to the macro-cell and the smallcells, respectively. In addition, we assume that there are $K_{0}$ macro-cell UEs (MUEs) and $K_{l}$ small-cell UEs (SUEs). For simplicity, the index of the UEs (MUEs and SUEs) associated with cell $l \in \mathcal{L}$ is denoted with $\mathcal{K}_{l}$. The HCN total available spectrum, $W_{\text {tot }}$, is divided into $N_{\text {tot }}$ subcarriers with each having a bandwidth of $W_{C}=\frac{W_{t o t}}{N_{t o t}}$. Specifically, the set of all accessible frequencies is denoted with $\mathcal{N}$ (where $|\mathcal{N}|=$ $N_{\text {tot }}$ ). We consider exclusive channel assignment, where any OFDMA subcarrier can only be employed by at most one UE in a given cell at a given time. Note that the UE-BS association is considered fixed during runtime.

The channel power gain from the cell- $m$ BS to the cell- $l k$ th UE over subcarrier $n$ is denoted with $h_{[k, l, m]}^{n}$. The received signal-to-interference-plus-noise ratio (SINR) at the cell- $l k$-th 
UE over subcarrier $n$ can be formulated as [9]

$$
\gamma_{[k, l]}^{n}=\frac{h_{[k, l, l]}^{n} p_{l}^{n}}{\sum_{m \in \mathcal{L} \backslash\{l\}} h_{[k, l, m]}^{n} p_{m}^{n}+\sigma_{[k, l]}^{n}}
$$

where $p_{l}^{n}$ is the transmit power of the cell-l BS over subcarrier $n$ and $\sigma_{[k, l]}^{n}$ is the noise power at the cell- $l k$-th UE over subcarrier $n$. We thus can express the rate for the cell- $l k$-th UE over subcarrier $n$ and the total throughput in cell- $l$ as

$$
\begin{aligned}
r_{[k, l]}^{n} & =W_{C} \log _{2}\left(1+\gamma_{[k, l]}^{n}\right) . \\
C_{l} & =\sum_{n \in \mathcal{N}} \sum_{k \in \mathcal{K}_{l}} \rho_{[k, l]}^{n} r_{[k, l]}^{n}
\end{aligned}
$$

where $\rho_{[k, l]}^{n} \in\{1,0\}$ indicates whether or not the $n^{\text {th }}$ subcarrier is assigned to the $\mathrm{UE}[k, l]$. Considering that BSs are the dominant sources of energy consumption in cellular networks, we approximate the $\mathrm{HCN}$ overall power consumption using the following linear power model [10]

$$
P=\zeta P_{T}+P_{C}
$$

where $\zeta, P_{T}$ and $P_{C}$ denote the BS reciprocal of drain efficiency of the power amplifier, transmission power, and circuit power consumption, respectively. Motivated by the approach in [8], the circuit power consumption is considered to be proportional to the total utilized bandwidth for transmission. Consequently, the total circuit power can be written as

$$
P_{c}=P_{s}+\gamma W
$$

where $P_{s}$ is the static circuit power in transmission mode and $\gamma$ is a constant corresponding to the dynamic power consumption per unit bandwidth. As a result, the total power consumption in the two-tier OFDMA-based $\mathrm{HCN}$ is defined as

$$
P=\sum_{l \in \mathcal{L}}\left(\zeta P_{T}^{[l]}+P_{C}^{[l]}\right)
$$

where $P_{T}^{[l]}$ and $P_{C}^{[l]}$ are the transmission power and the circuit power at the cell- $l$ BS.

Recall that EE is defined as the total number of successfully delivered bits per unit energy. The two-tier OFDMA-based HCN EE in the downlink can hence be expressed as

$$
\lambda_{E E} \triangleq \frac{\sum_{l \in \mathcal{L}} C_{l}}{\sum_{l \in \mathcal{L}}\left(\zeta P_{T}^{[l]}+P_{C}^{[l]}\right)}
$$

Here, we are concerned with the problem of achieving high EE whilst guaranteeing the required QoS constraints in each cell under limited bandwidth and transmit power resources. Hence, we formulate an optimization problem for maximizing EE under a series of (minimum) throughput requirements and maximum power budgets. Accordingly, the EE optimization problem for the two-tier OFDMA-based HCN is given by

$$
\begin{array}{ll}
\max _{[k, l]}^{n}, p_{l}^{n} & \lambda_{E E} \\
\text { s.t. } & \sum_{n \in \mathcal{N}} p_{l}^{n} \leq P_{\text {max }}^{[l]}, \forall l \in \mathcal{L}, \\
& \sum_{n \in \mathcal{N}} \sum_{k \in \mathcal{K}_{l}} \rho_{[k, l]}^{n} r_{[k, l]}^{n} \geq \delta_{\text {small }}, \quad \forall l \in \mathcal{L} \backslash\{0\},
\end{array}
$$

$$
\begin{aligned}
& \sum_{n \in \mathcal{N}} \sum_{k \in \mathcal{K}_{0}} \rho_{[k, 0]}^{n} r_{[k, 0]}^{n} \geq \delta_{\text {macro }}, \\
& \sum_{k \in \mathcal{K}_{l}} \rho_{[k, l]}^{n}=1, \forall n \in \mathcal{N}, \forall l \in \mathcal{L},
\end{aligned}
$$

where $P_{\max }^{[l]}$ is the maximum transmit power of the cell- $l$ BS and $\delta_{\text {macro }}\left(\delta_{\text {small }}\right)$ correspond to the UEs minimum throughput requirements in the macro-cell (small-cells), respectively. Therefore, constraints (9)-(11) are used to guarantee the maximum power budget and the minimum throughput target in each cell. In addition, the constraint in (12) corresponds to the exclusive subcarrier assignment strategy in any cell.

The EE optimization problem here, which considers join subcarrier assignment and power allocation in the presence of inter-cell interference, is mixed-combinatorial and non-convex. The solution is therefore nontrivial and cannot be obtained directly. As a result, in the following sections, we develop two different resource allocation approaches considering both spectrum underlay and overlay access.

\section{EE OPTIMIZATION IN OFDMA-BASED HCNS WITH SPECTRUM UNDERLAY ACCESS}

With spectrum underlay access, the small-cells share the available radio spectrum with the macro-cell and hence introduce inter-tier interference which renders the resource allocation problem significantly more challenging to tackle. In addition, the variables for subcarrier assignment and power allocation are coupled together and hence the non-convex optimization problem in (8)-(12) is extremely difficult to solve. In this section, we provide a fundamental study for energy-efficient design in OFDMA-based underlay HCNs. In particular, a relationship between optimal EE and achievable throughput is derived as in the following theorem.

Theorem I. For any rate vector for the macro-cell and the small-cells that satisfies the minimum throughput constraint, $\boldsymbol{C} \geq \boldsymbol{\delta}$, achieved with subcarrier assignment $\rho_{[k, l]}^{n}$ and power allocation $p_{l}^{n}$, the maximum achievable EE, namely,

$$
\begin{aligned}
& \lambda_{E E}^{*}(\mathbf{C}) \triangleq \max _{\rho_{[k, l]}^{n}, p_{l}^{n}} \lambda_{E E} \\
& \text { s.t. } \quad \sum_{n \in \mathcal{N}} p_{l}^{n} \leq P_{\text {max }}^{[l]}, \forall l \in \mathcal{L}, \\
& \mathbf{C} \geq \boldsymbol{\delta}, \forall l \in \mathcal{L}, \\
& \sum_{k \in \mathcal{K}_{l}} \rho_{[k, l]}^{n}=1, \forall n \in \mathcal{N}, \forall l \in \mathcal{L},
\end{aligned}
$$

where $\boldsymbol{C}=\left[\begin{array}{llll}C_{0} & C_{1} & \cdots & C_{L}\end{array}\right]$ and $\boldsymbol{\delta}=\left[\begin{array}{l}\delta_{\text {macro }} \\ \delta_{\text {small }}\end{array}\right.$ $\left.\delta_{\text {small }} \cdots \delta_{\text {small }}\right]$, is strictly quasi-concave in $\boldsymbol{C}$.

Therefore, Theorem I indicates that there always exists a unique $\mathrm{EE}$ solution. On the other hand, the original $\mathrm{EE}$ optimization problem is very challenging to solve due to the multiple inequality constraints in (10)-(11). By extending the Lagrange dual decomposition method for single-cell multicarrier systems [11] to our OFDMA-based underlay $\mathrm{HCN}$ setup, the gradient ascent approach can be invoked to generate $\mathbf{C}, l=0,1,2, \cdots$, and

$$
\mathbf{C}(n+1)=\left[\mathbf{C}(n)+\mu \nabla \lambda_{E E}(\mathbf{C}(n))\right]^{+} .
$$


However, a closed-form expression for the gradient of this vector does not exist, hence, it is impossible to employ (17) in order to solve (8)-(12). Nevertheless, we can transform the gradient ascent method in (17) using the following approach

$$
\begin{gathered}
C_{0}(n+1)=\left[C_{0}(n)+\mu \nabla \lambda_{E E}\left(C_{0}(n)\right)\right]^{+}, \\
\vdots \\
C_{L}(n+1)=\left[C_{L}(n)+\mu \nabla \lambda_{E E}\left(C_{L}(n)\right)\right]^{+} .
\end{gathered}
$$

As a result, the vector gradient can be alternatively decomposed into multiple scalar gradient, thus making the optimization problem relatively easier to solve. Accordingly, we propose an iterative resource allocation scheme to tackle the EE optimization problem for two-tier OFDMA-based HCNs. Similar to the gradient decomposition approach, by keeping one minimum throughput constraint at a time and setting all others as equality constraints, the problem with multiple inequality constraints can be decomposed into a series of optimization problems with single inequality constraint. Specifically, all other cells will be under equality constraints when cell- $l^{*}$ is under inequality constraint (minimum throughput requirement). Therefore, the EE problem is transformed into

$$
\begin{array}{ll}
\max _{\rho_{[k, l]}^{n}, p_{l}^{n}} & \lambda_{E E} \\
\text { s.t. } & C_{l}=\bar{C}_{l}, \forall l \in \mathcal{L} \backslash\left\{l^{*}\right\}, \\
& \sum_{n \in \mathcal{N}} \sum_{k \in \mathcal{K}_{l}^{*}} \rho_{\left[k, l^{*}\right]}^{n} r_{\left[k, l^{*}\right]}^{n} \geq \delta_{l}^{*}, \\
& \sum_{k \in \mathcal{K}_{l}} \rho_{[k, l]}^{n}=1, \forall n \in \mathcal{N}, \forall l \in \mathcal{L},
\end{array}
$$

where $\bar{C}_{l}$ represents the optimal throughput (for all other cells apart from cell $l^{*}$ ) obtained from the previous iteration. Under this setup, we can obtain the solution for cell $l^{*}, \bar{C}_{l^{*}}$ by solving the above single inequality constrained optimization problem. Once we obtain the updated throughput $\bar{C}_{l^{*}}$, the next cell is placed under inequality constraint (minimum throughput requirement), i.e., $\forall l^{*}+1 \in \mathcal{L}$, while all other cells have updated throughput values from the previous iteration. In particular, we can rewrite the constraints in (20)-(21) as

$$
\begin{gathered}
C_{l}=\bar{C}_{l}, \forall l \in \mathcal{L} \backslash\left\{l^{*}+1\right\}, \\
\sum_{n \in \mathcal{N}} \sum_{k \in \mathcal{K}_{l^{*}+1}} \rho_{\left[k, l^{*}+1\right]}^{n} r_{\left[k, l^{*}+1\right]}^{n} \geq \delta_{l^{*}+1} .
\end{gathered}
$$

The current maximum EE value is stored in the buffer and the corresponding optimal rate $\bar{C}_{l^{*}+1}$ for cell $l^{*}+1$ is updated. This process is repeated for all cells until convergence, i.e., $\lambda_{E E}^{o p t}(n+1)-\lambda_{E E}^{o p t}(n) \leq \varepsilon$. We provide a pseudocode for the proposed iterative resource allocation scheme:

(1) Initialize $l^{*}$ as the first cell in $\mathcal{L}$ with inequality constraint;

(2) Tackle the problem in (19)-(22) and store $\lambda_{E E}^{o p t}(n)$ in the buffer;

(3) Modify the constraints using (23)-(24) and update the corresponding rates;

(4) Repeat steps (2) and (3) until convergence $\lambda_{E E}^{o p t}(n+1)-$ $\lambda_{E E}^{o p t}(n) \leq \varepsilon$.

The decomposed EE optimization problem in (19)-(22) has a single inequality constraint. With a fundamental study of the problem, we can arrive at the following theorem.

Theorem II. The maximum EE achieved with a throughput for cell- $l^{*}, C_{l^{*}} \geq \delta_{l^{*}}$, subcarrier assignment $\rho_{[k, l]}^{n}$, and power allocation $p_{l}^{n}, \forall(l, n) \in(\mathcal{L}, \mathcal{N})$, namely,

$$
\begin{aligned}
& \lambda_{E E}^{*}\left(C_{l^{*}}\right) \triangleq \max _{\rho_{[k, l]}^{n}, p_{l}^{n}} \lambda_{E E} \\
& \text { s.t. } C_{l}=\bar{C}_{l}, \forall l \in \mathcal{L} \backslash\left\{l^{*}\right\}, \\
& \sum_{n \in \mathcal{N}} \sum_{k \in \mathcal{K}_{l}^{*}} \rho_{\left[k, l^{*}\right]}^{n} r_{\left[k, l^{*}\right]}^{n}=C_{l^{*}} \geq \delta_{l}^{*}, \\
& \sum_{k \in \mathcal{K}_{l}} \rho_{[k, l]}^{n}=1, \forall n \in \mathcal{N}, \forall l \in \mathcal{L}
\end{aligned}
$$

is strictly quasi-concave in $C_{l^{*}}$.

The function quasi-concavity property guarantees the existence of a unique maximum, hence Theorem II proves the existence of a unique EE solution. Moreover, the quasiconcavity of EE optimization problem further indicates that $\lambda_{E E}\left(C_{l^{*}}\right)$ either decreases or first increases and then decreases with $C_{l^{*}}$. Thus, problem (25)-(28) can be solved through a dual-layer decomposition method [12].

\section{Solution based on Spectrum Overlay Access AND RESOURCE EFFICIENCY}

The inter-tier interference from the proposed underlay-based approach may degrade EE especially under high throughput requirements in densely deployed scenarios. In addition, considering that the available bandwidth is fully exploited, a higher circuit power consumption and hence reduced EE performance may be incurred. Furthermore, although the proposed iterative resource allocation algorithm is numerically stable, its computational complexity depends on the number of optimizing variables, which can be large if the number of subcarriers or the number of UEs is large. Hence the complexity of this scheme is comparatively high. Based on the idea of spectrum overlay access and RE, we next develop a low-complexity solution for the two-tier HCN.

\section{A. Resource Efficiency Optimization for Macro-cell}

In [12], RE is defined as a weighted EE-SE trade-off using a normalizing factor $\beta$

$$
\lambda_{R E} \triangleq \frac{R}{P}\left(1+\beta \frac{\eta_{P}}{\eta_{W}}\right)
$$

where $\eta_{P}$ and $\eta_{W}$ respectively denote the power utilization and bandwidth utilization such that

$$
\eta_{P} \triangleq \frac{P}{P_{t o t}}, \quad \eta_{W} \triangleq \frac{W}{W_{t o t}} .
$$

We modify the RE to a more generalized expression

$$
\begin{aligned}
\lambda_{R E} & \triangleq \alpha \frac{R}{P}+(1-\alpha) \tau \frac{R}{P} \\
& =\frac{R}{P}\left(\alpha+(1-\alpha) \frac{\eta_{P}}{\eta_{W}}\right)
\end{aligned}
$$

where $0 \leq \alpha \leq 1$ and $\tau=\frac{W_{t o t}}{P_{t o t}}$. The generalized RE optimization problem in the downlink of the macro-cell can 
be mathematically formulated as

$$
\begin{aligned}
& \max _{\boldsymbol{\rho}, \mathbf{p}, \alpha} \frac{\sum_{k \in \mathcal{K}_{0}} \sum_{n \in \mathcal{N}} \rho_{[k, 0]}^{n} r_{[k, 0]}^{n}}{\zeta P_{T}+P_{C}}\left(\alpha+(1-\alpha) \frac{\eta_{P}}{\eta_{W}}\right) \\
& \text { s.t. } \quad \sum_{n \in \mathcal{N}} \sum_{k \in \mathcal{K}_{0}} r_{[k, 0]}^{n} \geq \delta_{0}, \\
& \sum_{k \in \mathcal{K}_{0}} \rho_{[k, 0]}^{n}=1, \forall n \in \mathcal{N}, \\
& \sum_{n \in \mathcal{N}} \sum_{k \in \mathcal{K}_{0}} \rho_{[k, 0]}^{n} \leq N_{t o t}, \\
& \sum_{n \in \mathcal{N}} p_{0}^{n} \leq P_{\text {max }}^{[0]}
\end{aligned}
$$

where $P_{T}=\sum_{n \in \mathcal{N}} p_{0}^{n}$ and $P_{C}=P_{s}+$ $\gamma W_{C} \sum_{k \in \mathcal{K}} \sum_{n \in \mathcal{N}} \rho_{[k, 0]}^{n}$. Problem (32)-(36) is mixedcombinational and non-convex. In order to tackle this, the subcarrier assignment and power allocation procedures are separated. Specifically, we first analyze the fundamental properties of the case with a given weight $\alpha$ and a given subcarrier assignment set. The findings are summarized in the following theorem. Note that for simplicity, here, we remove the index from the macro-cell parameters, e.g., MUEs set is changed from $\mathcal{K}_{0}$ to $\mathcal{K}$.

Theorem III. Considering a given weight $\alpha$, subcarrier allocation vector $\rho$ and its corresponding UEs set $\mathcal{S}_{k}(\forall k \in \mathcal{K})$, the maximum $R E$ at a certain transmit power, $P_{T}$, namely,

$$
\lambda_{R E}\left(P_{T}\right) \triangleq \max _{p_{k, n} \geq 0} \frac{\sum_{k \in \mathcal{K}} \sum_{n \in \mathcal{S}_{k}} r_{k, n}}{\zeta P_{T}+P_{C}}\left(\alpha+(1-\alpha) \frac{\eta_{P}}{\eta_{W}}\right)
$$

subject to

$$
\begin{gathered}
\sum_{n \in \mathcal{N}} \sum_{k \in \mathcal{S}_{k}} r_{k, n} \geq C_{0} \\
\sum_{k \in \mathcal{K}} \sum_{n \in \mathcal{S}_{k}} p_{k, n}=P_{T} \leq P_{\text {max }}
\end{gathered}
$$

has the following properties:

(i) $\lambda_{R E}\left(P_{T}\right)$ is a continuously differentiable quasi-concave function with respect to $P_{T}$,

(ii) the derivative of $\lambda_{R E}\left(P_{T}\right)$ meets the following condition

$$
\frac{d \lambda_{R E}\left(P_{T}\right)}{d P_{T}}=\frac{\left(\alpha+(1-\alpha) \frac{\eta_{P}}{\eta_{W}}\right) \frac{d \bar{R}\left(P_{T}\right)}{P_{T}}-\alpha \zeta \lambda_{E E}\left(P_{T}\right)}{\zeta P_{T}+P_{C}}
$$

where

$$
\begin{aligned}
\lambda_{E E}\left(P_{T}\right) & =\frac{\bar{R}\left(P_{T}\right)}{\zeta P_{T}+P_{C}} \\
\bar{R}\left(P_{T}\right) \triangleq \max _{p_{k, n} \geq 0} R\left(P_{T}\right) & =\max _{p_{k, n} \geq 0} \sum_{k \in \mathcal{K}} \sum_{n \in \mathcal{S}_{k}} r_{k, n}
\end{aligned}
$$

represents the maximum sum rate under the maximum power constraint and minimum rate constraint (38)-(39) meeting the condition

$$
\frac{d \bar{R}\left(P_{T}\right)}{P_{T}}=\max _{k \in \mathcal{K}, n \in \mathcal{S}_{k}} \frac{W_{C} g_{k, n} \log _{2} e}{1+p_{k, n}^{*} g_{k, n}}
$$

where $g_{k, n} \triangleq \frac{\left|h_{k}^{n}\right|^{2}}{\sigma_{k}^{n}}$ and $p_{k, n}^{*}\left(n \in \mathcal{S}_{k}\right)$ are respectively representing the channel-power-to-noise ratio $(C N R)$ of the $k$-th UE on the n-th OFDMA subcarrier and the optimal allocated power on the $n$-th subcarrier to obtain $\bar{R}\left(P_{T}\right)$.

For the case with fixed transmission power $P_{T}$ and subcarrier assignment vector $\rho$, we can rewrite the RE of the two-tier $\mathrm{HCN}$ as

$$
\lambda_{R E}\left(P_{T}\right)=\max _{p_{k, n} \geq 0} R\left(\frac{\alpha}{P}+(1-\alpha) \frac{W_{t o t}}{W P_{t o t}}\right)=\omega \bar{R}\left(P_{T}\right),
$$

where $\omega \triangleq \frac{\alpha}{P}+(1-\alpha) \frac{W_{t o t}}{W P_{t o t}}$. To derive the optimal power allocation, we can extend the multi-level water-filling scheme [12] to a HCN scenario as follows

$$
\begin{gathered}
\tilde{p}_{k, n}=\left(\mu_{k}-\frac{1}{g_{k, n}}\right)^{+}, \forall n \in \mathcal{S}_{k}, \\
\sum_{n \in \mathcal{S}_{k}, \tilde{p}_{k, n}>0} \sum_{k \in \mathcal{K}} W_{C} \log _{2}\left(\mu_{k} g_{k, n}\right)=C_{0} \\
p_{k, n}^{*}=\tilde{p}_{k, n}+\left(\mu-\frac{1}{g_{k, n}}-\tilde{p}_{k, n}\right)^{+} \\
\sum_{k \in \mathcal{K}} \sum_{n \in\left\{\mathcal{S}_{k} \mid \bar{p}_{k, n}>\tilde{p}_{k, n}\right\}}\left(\mu-\frac{1}{g_{k, n}}-\tilde{p}_{k, n}\right)=P_{T}-\sum_{k \in \mathcal{K}} \sum_{n \in \mathcal{S}_{k}} \tilde{p}_{k, n},
\end{gathered}
$$

where $\mu_{k}$ and $\mu$ are used to denote the intermediate variables. The multi-level water-filling approach consists of two steps. Firstly, the power is allocated in order to satisfy the minimum rate requirement of the macro-cell UEs, where the allocated power in this step is $P_{S}=\sum_{k \in \mathcal{K}} \sum_{n \in \mathcal{S}_{k}} \tilde{p}_{k, n}$. Next, the remaining power is allocated in order to further improve the sum rate. Since quasi-concave function guarantees the existence of a unique maximum, we thus apply the gradient method to search for the optimal power. In particular, for a fixed subcarrier assignment set, gradient-based power adaptation can be used with single-UE water-filling in (45)-(46), and the multi-level water-filling in (47)-(48), where the power is updated using the gradient of RE

$$
P_{T}(n)=P_{T}(n-1)+t \times \frac{d \lambda_{R E}\left(P_{T}\right)}{d P_{T}}
$$

with $t$ being the step size. Since the quasi-concavity property implies that $\lambda_{R E}\left(P_{T}\right)$ either strictly decreases or first increases and then strictly decreases with $P_{T}$, the proposed algorithm will terminate with either convergence or $P_{0}=C_{0}^{-1}\left(\mathcal{S}_{k}, \gamma_{k}\right)$ if $\lambda_{R E}\left(P_{T}\right)$ is monotonically decreasing in $\left[P_{0}, P_{\max }\right]$ and $P_{\max }$ if $\lambda_{R E}\left(P_{T}\right)$ is monotonically increasing in $\left[P_{0}, P_{\text {max }}\right]$.

We are now ready to investigate the subcarrier assignment strategy. We assign each subcarrier to the MUE that would achieve the highest SINR on that subcarrier.

$$
\rho_{k}^{n *}=\left\{\begin{array}{l}
1, \text { if } k=\arg \max _{k \in \mathcal{K}} \gamma_{k}^{n} \\
0, \text { otherwise }
\end{array} .\right.
$$

Note that the proposed power allocation approach only aims to solve the RE optimization problem (32)-(36) with fixed normalizing factor $\alpha$. Let $\lambda_{R E}(\alpha)$ denote the objective value of problem (32)-(36) with a given $\alpha$. It is easy to see that $\lambda_{R E}(1)$ denotes the maximum RE value of problem (32)-(36) that only aims to maximize EE without taking SE into account. On the other hand, $\lambda_{R E}(0)$ corresponds to the case in which 
SE is maximized without taking EE into account. Therefore, based on this result, we further develop a bi-section approach to numerically search for the optimal value of $\alpha$.

By employing the proposed bi-section based subcarrier assignment and power allocation approach, the optimal RE can be obtained for a given bandwidth (given number of subcarriers). As a result, starting from $W_{\min }=K W_{C}$ (i.e., each UE should be guaranteed at least one subcarrier), we apply the proposed gradient-based power adaptation and the subcarrier allocation policy to the current bandwidth setting, and store the optimal RE value in the buffer $\lambda_{R E}(W)$. Then, we increment the bandwidth using $W=W+W_{C}$. The proposed subcarrier assignment and power allocation approach is performed again to obtain the maximum RE of the macrocell under the updated bandwidth. This procedure is repeated for all possible bandwidth options, i.e., from $W_{\min }$ to $W_{t o t}$. Hence, the optimal RE and the corresponding bandwidth of the macro-cell is determined. The remaining bandwidth is then dedicated to the small-cell operation.

\section{B. Energy-Efficiency Optimization for Small-Cells}

Once the RE of the macro-cell is maximized, the remaining bandwidth is allocated exclusively to the small-cells. Since the macro-cell and small-cells are not sharing the same spectrum, there is no inter-tier interference. Moreover, considering the maximum transmit power of the small-cells is usually low (small coverage), and small-cells are geographically separated, the intra-tier interference between small-cells is considerably small. Therefore, considering the intra-tier interference as noise, we propose a low-complexity suboptimal resource allocation approach to maximize the EE of the small-cells.

Under this overlay-based setup, intra-tier interference is suppressed. The SINR expression can thus be rewritten as

$$
\gamma_{[k, l]}^{n}=\frac{h_{[k, l, l]}^{n} p_{l}^{n}}{\sigma_{[k, l]}^{n}} .
$$

As a result, the optimization problem in (8)-(12) can be decomposed to a series of relatively isolated and simple optimization problems. In other words, one only needs to solve the EE maximization problem for each small-cell $(l \in \mathcal{L})$. This can be formulated as

$$
\begin{array}{ll}
\max _{\rho_{[k, l]}^{n}, p_{l}^{n}} & \frac{C_{l}}{\zeta P_{T}^{[l]}+P_{C}^{[l]}} \\
\text { s.t. } & \sum_{n \in \mathcal{N}} \sum_{k \in \mathcal{K}_{l}} p_{l}^{n} \leq P_{\text {max }}^{[l]}, \\
& \sum_{n \in \mathcal{N}} \sum_{k \in \mathcal{K}_{l}} \rho_{[k, l]}^{n} r_{[k, l]}^{n} \geq \delta_{\text {small }}, \\
& \sum_{k \in \mathcal{K}_{l}} \rho_{[k, l]}^{n}=1, \forall n \in \mathcal{N}
\end{array}
$$

The EE maximization problem in (52)-(55) is a special case of the RE maximization problem in (32)-(36), and hence we apply the proposed subcarrier allocation policy in (50) for the remaining bandwidth, and then perform the multi-level waterfilling algorithm in (45)-(48) to obtain the optimal value. This procedure is repeated for all the small-cells.

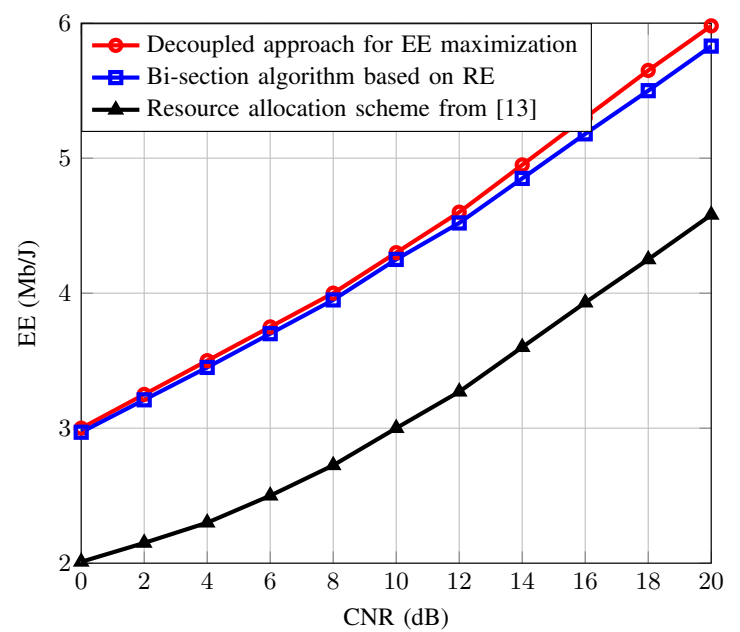

Fig. 1: Comparison of different subcarrier assignment and power allocation schemes in terms of EE.

\section{Simulation Results}

In this section, we present numerical results in order to verify our theoretical findings and analyze the performance of the proposed underlay and overlay approaches in terms of EE. It is assumed that ten uniformly-distributed small-cells are in the coverage area of a existing macro-cell, where ten and three uniformly-distributed UEs are serviced in the macro-cell and each small-cell, respectively. The radius of the macro-cell is set to $250 \mathrm{~m}$, and that of the small-cells is set to $50 \mathrm{~m}$. It should be noted that all results are obtained from various random locations of the UEs with identical and independent Rayleigh fading channels. The minimum throughput requirements for macro-cell and small-cells are set to $100 \mathrm{Mbps}$.

In the first simulation, we evaluate the EE performance of the different schemes with spectrum underlay and overlay access. For comparison purposes, we compare the proposed schemes against the joint subcarrier assignment and power allocation scheme in [13]. The optimal EE is evaluated across the 0-20 dB CNR range. As it can be seen from Fig. 1, the EE achieved by the proposed overlay-based approach is very close to that of the proposed underlay-based approach whilst being much more efficient in terms of computational complexity. It is important to highlight, however, that the performance gap increases in high CNR region. This is because the overlaybased approach treats the intra-tier interference as noise, and will become dominant when the noise power diminishes at high CNR regime; hence resulting in reduced EE performance. Furthermore, both algorithms achieve higher EE compared to the scheme in [13] which aims to maximize the sum rate.

The impact of the number of subcarriers on the optimal EE is illustrated in Fig. 2. It can be seen that with a moderate number of subcarriers (small bandwidth), e.g., $N \leq 1000$, the EE achieved by the proposed overlay-based approach is lower than that of the proposed approach using underlay transmission. However this trend is reversed when the system has a larger bandwidth (e.g., $N \geq 1000$ ). The reason for this observation is that the proposed underlay-based approach allocates all available bandwidth to all cells. The excessive transmissionassociated circuit power, which is modeled as a linear function 


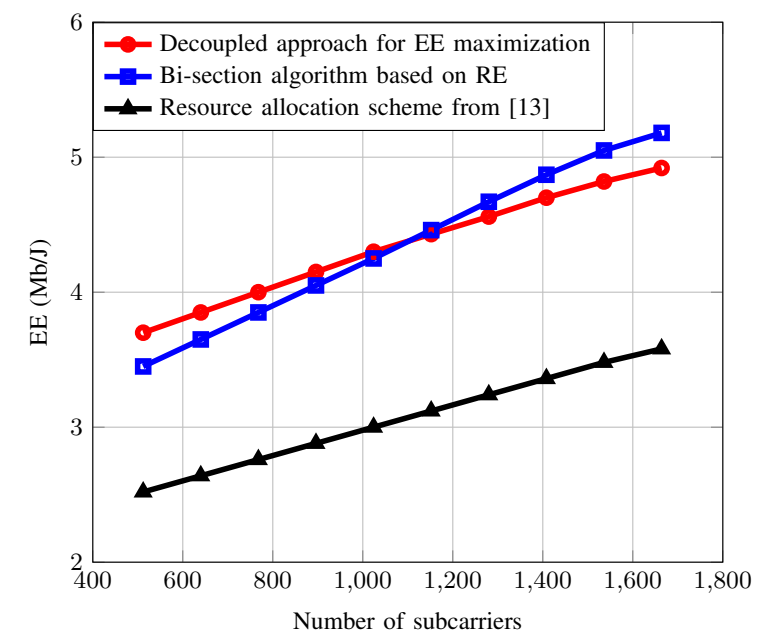

Fig. 2: Impact of the number of subcarriers on the EE performance.

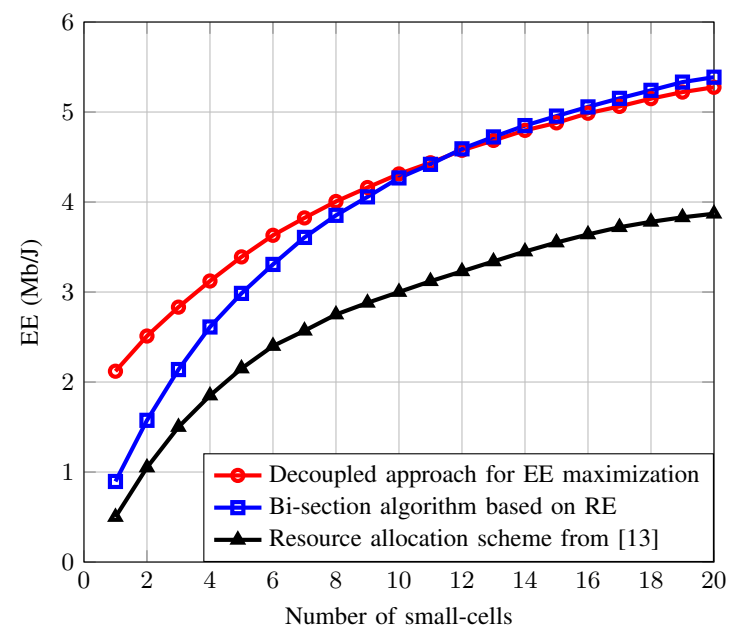

Fig. 3: Impact of small-cells density on the EE performance.

of the bandwidth, will reduce the EE performance in a system with large bandwidth. On the other hand, the proposed overlaybased approach allocates the exclusive spectrum parts to the macro-cell and small-cells and hence is more suitable for in the context of bandwidth-abundant HCNs.

Finally, the impact of small-cells density on the optimal $\mathrm{EE}$ is investigated in Fig. 3. As shown in the figure, with a lower small-cells density, e.g., $L \leq 10$, the EE achieved by the proposed bisection-based approach is lower than that of the proposed decomposed resource allocation approach. However, for the case of dense small-cells, e.g., $L \geq 10$, the $\mathrm{EE}$ achieved by the strategy using RE and overlay transmission is superior. This is because when the small-cells density is low, the inter-tier interference will have less impact resulting in lower transmit power levels needed to satisfy the QoS targets. Therefore, the underlay transmission strategy is suitable under this setup. On the other hand, for the case of a dense deployed HCN, e.g., with $L \geq 10$, the inter-tier interference will become significant when spectrum is shared by the different tiers. Hence, extra power is required to maintain the throughput requirements of the UEs. Moreover, since macro-cell and small-cells occupy the whole bandwidth at the same time, the excessive transmission associated circuit power will further degrade EE performance. Consequently, the proposed overlaybased approach where different portions of the spectrum is allocated exclusively to the macro-cell and the small-cells is more suitable for dense multi-tier cellular environments.

\section{Conclusions}

In this paper, we have addressed the EE optimization problem for OFDMA-based two-tier HCNs consisting of a macro-cell and multiple small-cells. Considering underlay transmission, where macro-cell and small-cells are sharing the available spectrum, we proved the relationship between EE and achievable rate is a quasi-concave function. On the basis of this property, we decomposed the original problem with multiple inequality constraints into multiple optimization problems with single inequality constraints, which can be efficiently solved. On the other hand, the underlay approach may not be energyefficient due to severe inter-tier interference in a dense HCN scenario. In addition, it will lead to a higher power consumption in a bandwidth-abundant system and hence reduce the EE performance. Therefore, we developed a novel lowcomplexity resource allocation scheme based on the idea of overlay transmission and RE. Simulation results confirmed the theoretical findings and demonstrated that the proposed algorithms can efficiently approach the optimal EE.

\section{REFERENCES}

[1] J. G. Andrews, "Seven ways that HetNets are a cellular paradigm shift," IEEE Commun. Mag., vol. 51, no. 3, pp. 136-144, Mar. 2013.

[2] D. Lopez-Perez, A. Valcarce, G. de la Roche, and J. Zhang, "OFDMA femtocells: A roadmap on interference avoidance," IEEE Commun. Mag., vol. 47, no. 9, pp. 41-48, Sep. 2013.

[3] M. Kang, M. S. Alouini, and L. Yang, "Outage probability and spectrum efficiency of cellular mobile radio systems with smart antennas," IEEE Trans. Commun., vol. 50, no. 7, pp. 1871-1877, Dec. 2002.

[4] H. Dai and H. Poor, "Asymptotic spectral efficiency of multi-cell MIMO systems with frequency-flat fading," IEEE Trans. Signal Process., vol. 51, pp. 2976-2988, Nov. 2003.

[5] G. Lim, C. Xiong, L. J. C. Jr., and G. Y. Li, "Energy-efficient resource allocation for OFDMA-based multi-RAT networks," IEEE Trans. Wireless Commun., vol. 13, no. 5, pp. 2696- 2705, May 2014.

[6] S. Bu, F. Yu, and $\mathrm{H}$. Yanikomeroglu, "Interference-aware energyefficient resource allocation for OFDMA-based heterogeneous networks with incomplete channel state information," IEEE Trans. Veh. Tech., vol. 64, no. 3, pp. 1036-1050, Mar. 2015.

[7] W. S. Lai, T. H. Chang, and T. S. Lee, "Joint power and admission control for spectral and energy efficiency maximization in heterogeneous OFDMA networks," IEEE Trans. Wireless Commun., vol. 15, no. 5, pp. 3531- 3547, May 2016.

[8] S. Zhang, Y. Chen, and S. Xu, "Joint bandwidth-power allocation for energy efficient transmission in multi-user systems," in Proc. IEEE Globecom Workshops, Miami, FL, Dec. 2010.

[9] A. Shojaeifard, K. A. Hamdi, E. Alsusa, D. K. C. So, and J. Tang, "Exact SINR statistics in the presence of heterogeneous interferers," IEEE Trans. on Inf. Theory, vol. 61, no. 12, pp. 6759-6773, Dec 2015.

[10] S. Cui, A. Goldsmith, and A. Bahai, "Energy-constrained modulation optimization," IEEE Trans. Wireless Commun., vol. 4, no. 5, pp. 23492360, Sep. 2005.

[11] C. Xiong, G. Li, S. Zhang, Y. Chen, and S. Xu, "Energy- and spectralefficiency tradeoff in downlink OFDMA networks," IEEE Trans. Wireless Commun., vol. 10, no. 11, pp. 3874-3886, Nov. 2011.

[12] J. Tang, D. K. C. So, E. Alsusa, and K. A. Hamdi, "Resource efficiency: A new paradigm on energy efficiency and spectral efficiency tradeoff," IEEE Trans. Wireless Commun., vol. 13, no. 8, pp. 46564669, Aug. 2014.

[13] D. T. Ngo, S. Khakurel, and T. Le-Ngoc, "Joint subchannel assignment and power allocation for OFDMA femtocell networks," IEEE Trans. Wireless Commun., vol. 13, no. 1, pp. 342-355, Jan. 2014. 